\title{
LA INCORPORACIÓN DE LAS TIC EN EL DESARROLLO HISTÓRICO DE UNA LICENCIATURA EN EDUCACIÓN
}

\section{THE INCORPORATION OF ICT IN THE HISTORICAL DEVELOPMENT OF A BACHELOR'S DEGREE IN EDUCATION}

\section{Karla Lariza Parra Encinas ${ }^{1}$ \\ Arnoldo Lizárraga Juárez²}

Universidad Autónoma de Baja California

\section{RESUMEN}

En este trabajo se presenta una revisión histórica de las diversas funciones que han cumplido las TIC a lo largo del desarrollo de la Licenciatura en Ciencias de la Educación de la Universidad Autónoma de Baja California (UABC), desde sus inicios hasta la creación del Centro de Educación Abierta y a Distancia. La revisión fue realizada con base en una investigación documental de los archivos de la UABC en general y de la

1 Docente investigadora Universidad Autónoma de Baja California parra.karla@uabc.edu.mx https://orcid.org/0000-0002-2855-2224

2 Docente investigador Universidad Autónoma de Baja California arnoldo.lizarraga@uabc.edu.mx https://orcid.org/0000-0003-4228-7823

Mexicali, Baja California, México
Facultad de Ciencias Humanas en lo particular, y se enmarca en los contextos institucional y nacional a través del modelo educativo de la UABC, los Planes Nacionales de Desarrollo de México y los Planes Sectoriales de Educación.

PALABRAS CLAVE: TIC, Ciencias de la Educación, Educación Superior.

\section{ABSTRACT}

This paper presents a historical review of the various functions that ICTs have fulfilled throughout the development of the Bachelor of Education Sciences of the Autonomous University of Baja California (UABC), from its inception to the creation of the Center for Open and Distance Education. The review was based 
on a documentary research of the archives of the UABC in general and the Faculty of Human Sciences in particular, and is framed in the institucional and national contexts through the educational model of the UABC, the National Development Plans of México and the Sectoral Education Plans.

Keywords: ICT, Education Sciences, Higher Education

\section{INTRODUCCION}

En el presente trabajo se realiza una revisión sobre las funciones que han desempeñado las Ilamadas Tecnologías de la Información y Comunicación (TIC) a lo largo del desarrollo de la Licenciatura en Ciencias de la Educación, de la Facultad de Ciencias Humanas (FCH) de la Universidad Autónoma de Baja California (UABC). Cabe destacar que dichas tecnologías han sido incorporadas de acuerdo con diversos fines dentro de esta carrera: como herramientas de enseñanza y aprendizaje integradas curricularmente a procesos escolarizados, semiescolarizados y no escolarizados o en línea; como contenido o parte del objeto de estudio de la carrera, es decir, no solo como apoyos didácticos, sino también como parte fundamental del perfil profesional de egreso de sus estudiantes; como herramientas de apoyo a la investigación y, por último, como herramientas de apoyo a los procesos administrativos.

En dicha revisión se esclarecerá el papel que han jugado estas herramientas tecnológicas desde los inicios de la licenciatura, cuando en un aula equipada como laboratorio de química funcionaba un pequeño taller de fotografía, donde a los alumnos de la carrera de educación se les enseñaba a realizar audiovisuales con diapositivas y a trabajar la fotografía en blanco y negro con fines didácticos, hasta la llegada de Internet y de nuevos y modernos talleres, aulas de medios y laboratorios equipados con tecnología de última generación, pasando por la creación de una moderna y funcional biblioteca totalmente equipada y terminando con la creación de una extensión del Centro de Educación Abierta y a Distancia al interior de la Facultad, a partir del cual arrancan formalmente los cursos en línea mediante la plataforma oficial de la UABC.

Además de los mencionados anteriormente, algunos acontecimientos que marcaron etapas importantes en el uso de las tecnologías fueron, entre otros, los modelos educativos adoptados e implementados por la UABC, la instauración del Plan de Estudios Flexible y por Competencias y la modalidad del Plan de Estudios Semiescolarizada. Todo ello en consonancia con políticas a nivel nacional de diversificación de la oferta educativa, de flexibilización curricular por competencias y de inclusión de las TIC en los procesos educativos.

\section{INTEGRACION DE LAS TECNOLOGIAS EN EL PROCESO EDUCATIVO}

Es imperativo que las políticas de inclusión y fortalecimiento del uso de las TIC en los procesos educativos sean consideradas y declaradas en el marco de las políticas educativas definidas en los Planes Nacionales de Desarrollo y en los Planes Nacionales de Educación (Consejo Federal de Educación, 2010).

Las políticas y procesos de incorporación de las TIC en las escuelas han presentado problemas en todos los países por diversos motivos. Uno de ellos tiene que ver con una característica particular que presenta el desarrollo de estas tecnologías en nuestras sociedades, específicamente la exagerada rapidez en que lo nuevo se convierte en obsoleto. Éste es uno de los problemas más importantes que los gobiernos, las instituciones en general y las educativas en particular, deben enfrentar en cuanto a las políticas de acceso a las tecnologías (Aguerrondo, 2006). Lo que es muy evidente es la necesidad de incluir las TIC en la realidad del 
sistema educativo en todos sus niveles como herramientas que apoyen tanto lo referido al aprendizaje de los alumnos y de los profesores, como contenido, como apoyo a las actividades de investigación y como herramientas para mejorar la eficiencia de la gestión de las instituciones y del sistema educativo en general. Es por ello que este proceso de incorporación de las TIC debe convertirse en una oportunidad para producir cambios profundos en la educación (Aguerrondo, 2006).

En el caso de México, la inclusión de las TIC en los procesos educativos se ha convertido desde hace ya varias décadas en una de las prioridades declaradas en los Planes Nacionales de Desarrollo, concebidas como herramientas fundamentales para la participación en la sociedad del conocimiento y como elemento clave para la consecución de sus metas, objetivos y estrategias prioritarias. Así por ejemplo, en el caso del Plan Nacional de Desarrollo (PND) 2013-2018, se encuentra el objetivo 3.1 que se refiere a "desarrollar el potencial humano de los mexicanos con educación de calidad", cuya estrategia 3.1.2 contempla el "modernizar la infraestructura y el equipamiento de los centros educativos", mientras que la estrategia 3.1.4 declara "promover la incorporación de las Nuevas Tecnologías de la Información y Comunicación en el proceso de enseñanzaaprendizaje" (Gobierno de la República, 2013: 123-124).

Este objetivo y estrategias del PND quedan cubiertos con el Objetivo 1 del Programa Sectorial de Educación 2013-2018, de la Secretaría de Educación Pública (SEP), que a la letra dice: "asegurar la calidad de los aprendizajes en la educación básica y la formación integral de todos los grupos de la población" y con el objetivo 2: "fortalecer la calidad y la pertinencia de la educación media superior, superior y formación para el trabajo, a fin de que contribuyan al desarrollo de México" (SEP, 2013: 37), según la tabla de alineación a las metas nacionales del mismo plan.

Así mismo, al hacer una revisión de los PND de los dos sexenios anteriores (Gobierno de la República, 2001; 2007) y de sus respectivos Programas de Educación (SEP, 2001; 2007), se hacen evidentes las diversas políticas de inclusión de las TIC en los procesos educativos. En consecuencia con éstos, se encuentra también el Plan Estatal de Desarrollo de Baja California 2014-2019 (Gobierno del Estado de BC, 2014), el Programa de Educación de Baja California 2015-2019 (Sistema Educativo Estatal, 2015), así como también los Planes de Desarrollo Institucional de la UABC (UABC, 2003; 2007; 2011b; 2015b). En todos ellos, la incorporación de las tecnologías en los procesos educativos es considerada como una de las piezas fundamentales que contribuyen a la formación integral del estudiante y al mismo tiempo a la transformación de México en una sociedad del conocimiento, por tanto, se establecen también los objetivos y estrategias necesarias para realizar tal acción.

La incorporación de las tecnologías a los procesos educativos es un fenómeno que se ha ido incrementando en los últimos años en los diferentes niveles de enseñanza del sistema educativo, dando respuesta a los cambios en el contexto de la sociedad de la información o sociedad del conocimiento (Nosiglia y Barboza, 2013). Esta incorporación constituye un reto a superar más en el plano pedagógico que el tecnológico. Con su introducción en las escuelas se prevé que haya cambios significativos en la estructura escolar, el papel del maestro y el de los propios estudiantes (Cabero, 2007).

De acuerdo a Ferreiro y De Napoli (2008), los retos que confrontan las instituciones educativas en su adopción de las TIC pueden sintetizarse en tres: el del acceso, el empleo y la integración, y pueden ilustrarse con las vocales A, E y la I. La A, 
referida a la necesidad de tener acceso (políticas y acciones de acceso a la tecnología), la E, referida al empleo, la disposición y capacitación que tengamos para el correcto uso de las TIC dentro del proceso de enseñanza y aprendizaje, mientras que la I alude a la integración de las tecnologías al desarrollo de los contenidos curriculares. Quizás el de integrar la tecnología con el contenido sea el reto pedagógico más importante (Ferreiro y De Napoli, 2008).

La preocupación sobre el tema de la integración curricular de las TIC surge al asumir que el proceso de acercamiento entre TIC y educación ha superado ya la barrera de acceder (A) a la tecnología y además se ha aprendido a utilizar por gran parte de los educadores y del alumnado (E), generando entonces el ambiente propicio para desarrollar la nueva etapa: ¿cómo integrar las TIC eficazmente al currículo? (I). Para responder dicha interrogante, es necesario conocer en primer lugar qué es lo que se sabe en este campo (Parra y Pincheira, s.f.).

Uno de los factores fundamentales que ha obstaculizado la utilización educacional de dichas tecnologías es la frecuente confusión entre usar las tecnologías y su integración curricular. Usar las tecnologías puede implicar utilizarlas para los más diversos fines, sin un propósito claro de apoyar el aprender de un contenido. Por el contrario, la integración curricular de las tecnologías de la información implica el uso de estas tecnologías para lograr un propósito en el aprender de un concepto, un proceso, un contenido, en una disciplina curricular específica. Esta integración implica e incluye necesariamente el uso curricular de las TIC (Sánchez, 2003)

Es necesario tomar conciencia de una vez por todas y adoptar una serie de medidas que vayan más allá de la dotación de ordenadores, para conseguir que la incorporación plena de las TIC a los centros educativos contribuya de manera decidida en la mejora del proceso de enseñanza y aprendizaje (Pariente, 2005).

De acuerdo con Sánchez (2003) "integrar las TICs al currículum implica integrarlas a los principios educativos y la didáctica que conforman el engranaje del aprender. Esto es, integrar curricularmente las TICs implica empotrarlas en las metodologías y la didáctica que facilitan un aprender del aprendiz" (p. 52). En consecuencia con esta definición, la integración de las TIC en la Carrera de Cs. de la Educación de la $\mathrm{FCH}$, debe ser en armonía con el modelo educativo vigente en la UABC.

En ese sentido, el modelo educativo de la UABC, se sustenta filosófica y pedagógicamente en el humanismo, que destaca la concepción del ser humano como una persona integral; el constructivismo, que promueve un aprendizaje activo y centrado en el alumno; y la educación a lo largo de la vida, que enfoca su atención en los aprendizajes, en vez de limitarse a la transmisión de conocimiento (UABC, 2013, p. 33).

Por lo tanto, el rol de las TIC en el proceso de enseñanza-aprendizaje no es el de intentar la instrucción de los estudiantes, ni reforzar viejas prácticas tradicionales o conductistas, sino, más bien, el de servir como herramientas colaborativas de construcción del conocimiento $\mathrm{y}$, al mismo tiempo, permitirles a los estudiantes desarrollar habilidades de orden superior, como la resolución de problemas, la creación de proyectos, el modelamiento de procesos que ellos ya manejan y usar el computador como herramienta para presentar la organización que su mente le da a tales eventos. De allí que a partir del currículum se analicen actividades que desarrollen habilidades de pensamiento $o$ sociales para apoyar el aprendizaje mediante recursos informáticos (Herrera, s.f.) 


\section{EL INICIO}

La historia del desarrollo de las TIC se inicia mucho antes de que las computadoras pasen a ser usadas domésticamente, pero en el campo de la educación se impone popularmente a principios de los 80's, cuando el precio de los microprocesadores las hace posibles para el mercado doméstico. La historia de la incorporación de estas tecnologías en la enseñanza puede rastrearse desde las máquinas de enseñar y la Enseñanza Asistida por Ordenador.

El ejercicio de la docencia contemporánea en el mundo en general y en Latinoamérica y el Caribe, se ha visto influenciado por el impacto de elementos que se derivan básicamente de dos grandes contextos disciplinarios. Por una parte, de las teorías educativas que critican a las prácticas instruccionales identificadas con los presupuestos conductistas $\mathrm{y}$, por otra, de los hallazgos de la investigación teórica que han permitido una contundente reflexión sobre la práctica docente a partir de los supuestos del paradigma cognitivo, las teorías del aprendizaje significativo, el aprendizaje social, el constructivismo y el aprendizaje colaborativo (Fernández, 2001).

Desde 1977, el desarrollo del Proyecto Ciencias de la Educación enfrentó una serie de problemáticas relacionadas con la naturaleza de su objeto de estudio y con la situación histórica y contextual de la que formó parte. La mínima investigación existente respecto al desarrollo curricular, aunada a la escasez de recursos y de personal especializado en esta área, provocó la conformación de sus planes y programas educativos bajo paradigmas tradicionales. No obstante, el proceso de desarrollo curricular del mencionado proyecto avanzó con todo y las limitantes existentes y, sin pretender obtener un producto acabado e infalible, se contó con un planteamiento teórico- metodológico e instrumental que le dio rumbo a la actividad formativa del Licenciado en Ciencias de la Educación. Tal planteamiento curricular pretendía responder a la problemática educativa que nuestra región y el país enfrentaban en esa época (UABC-ECE, 1983).

En ese sentido, el Plan de Estudios de la Licenciatura en Ciencias de la Educación 1978, emanado bajo estas circunstancias, tenía como objetivo general el de "formar profesionales a nivel licenciatura en Ciencias de la Educación capaces de satisfacer las necesidades educativas de la comunidad en las áreas de Planeación Educativa; Administración de Instituciones Educativas; Formación y Capacitación Docente y Extensión Educativa" (UABC-ECE, 1983: 9). Para el logro de este objetivo, desde 1978 la Escuela de Ciencias de la Educación, como se denominó en sus inicios, contaba con un laboratorio de química que nunca se utilizó como tal, sino como laboratorio audiovisual y de fotografía, en lo que fuera el salón 204 del edificio 200 y que en la actualidad alberga a las actividades de Servicio Social, Prácticas Profesionales, Titulación, Movilidad Académica y Estudiantil, etc. (UABC-ECE, 1983)

En 1980 surge la primera aula audiovisual, que consistía en un salón acondicionado (108) en donde se contó con equipo de televisión comprado al Canal 3 de Mexicali, consistente en una cámara de TV, grabadora de video de $3 / 4$ y monitor. Con esas limitaciones los alumnos de la carrera de Educación realizaban ciertas prácticas en sus materias relacionadas con la producción de materiales didácticos. Como se puede apreciar, el rol de las herramientas tecnológicas en un inicio fue el de reforzar la formación del estudiante, es decir, como "contenido u objeto de estudio" de la Licenciatura en Ciencias de la Educación. 


\section{EL EDIFICIO DE LOS 300}

Para 1982, los recursos tecnológicos disponibles para cubrir la función de apoyo a la docencia o de herramientas didácticas en la licenciatura eran escasos, por lo general la mayoría de los docentes solo utilizaban pizarrón y gis y algunos medios didácticos tradicionales como complemento a sus clases expositivas, bajo esquemas o modelos conductistas y/o tradicionales. Los dispositivos tecnológicos se reducían a algunos televisores, videocaseteras VHS y proyectores de acetatos y de diapositivas, así como también ciertos aparatos llamados cuerpos opacos u opascopios.

En este mismo año, se inauguró el edificio de aulas denominado "300", en donde se incluyó la primera sala audiovisual con capacidad para 100 personas y equipada con lo básico, esto es, un televisor, reproductor de VHS y posteriormente con reproductor de DVD. También se disponía de un equipo de sonido y micrófonos y, desde entonces, se ha utilizado para realizar eventos académicos y culturales. En la actualidad cuenta con una cabina de controles, equipo de sonido, cañón de video e internet inalámbrico, lo que permite que se brinde un espacio actualizado y de mayor calidad para eventos que contribuyen a enriquecer la formación integral del estudiante. Años después se inaugura el edificio "700", que incluye además de 8 aulas de clase, la segunda sala audiovisual que en la actualidad se denomina Aula Magna, con capacidad para 90 usuarios y equipada con sonido local, cañón de video, Internet inalámbrico, etc.

En cuanto a la función de la tecnología como contenido u objeto de estudio, es decir, como una parte medular en la formación del alumnado, se tuvo un considerable avance, pues en ese mismo edificio, en el salón 301 , se reubicó el laboratorio audiovisual que venía trabajando en el salón 108 y se crearon las áreas o salas de grabación de audio y video, de fotografía y de diseño gráfico. Estos espacios, aunque modestamente equipados, obedecían a las necesidades de prácticas académicas de materias como: Taller de Fotografía, Taller de Diseño Gráfico, Docencia y Métodos y Técnicas Audiovisuales, fundamentales en la formación de los educólogos (UABC-FCH, 1986).

En 1986, después de un proceso de evaluación curricular, surge el Plan de Estudios 1986-2, en donde a partir de sus objetivos curriculares y su perfil de egreso se evidencia un considerable énfasis en el estudio de los medios de comunicación educativa como contenido $u$ objeto de estudio de todo profesionista de la educación.

\section{ELEDIFICIODETALLERESYLABORATORIOS}

A partir del segundo semestre de 1986 , da inicio la Licenciatura en Ciencias de la Comunicación en la Facultad de Ciencias Humanas, que obedeció a la idea de formar profesionales en el área que vinieran a satisfacer las demandas que surgen a partir de entender a la comunicación como una pieza clave de las nuevas definiciones y configuraciones de la distribución de los saberes y competencias sociales y culturales (UABC-FCH, 2011).

Dada la naturaleza y objeto de estudio de esta licenciatura y después de varios años de que sus estudiantes realizaban sus prácticas de campo con las limitaciones de una inadecuada infraestructura y equipamiento, se inaugura en 1991 el edificio de Talleres y Laboratorios en la $\mathrm{FCH}$, en el que, en su planta baja, contó con equipos de última generación en las áreas de televisión, radio, fotografía e informática, mientras que su planta alta albergó a tres laboratorios de la Licenciatura en Psicología, misma que inició sus labores en 1985-2.

La construcción de dichos Talleres y Laboratorios, significó un gran salto para la calidad en la formación no solo para los alumnos de la carrera 
de Comunicación, sino también para los de las demás carreras, entre ellas, la de Educación. Este fue un acontecimiento que marcó un gran avance en las TIC incorporadas como contenido u objeto de estudio, puesto que en el Plan de Estudios vigente (1986-2) los estudiantes de materias como: Teoría de la Comunicación, Tecnología Educativa I, II y III y Producción de Medios Educativos (UABC-FCH, 1986), asistían a realizar sus prácticas formativas relacionadas con la producción de recursos didácticos, tales como grabaciones de audio y fotografía en diapositivas para sus audiovisuales, grabación y edición de videos, entre otros.

En cuanto a las TIC y su función como herramientas didácticas $\mathrm{o}$ de apoyo a la docencia, los Talleres y Laboratorios cuentan con un laboratorio de informática que alberga un aula de medios con capacidad para 35 estudiantes y otra con capacidad para 20. En estas aulas los alumnos de todas las carreras, incluyendo la de Educación, asisten a tomar clases de materias como: Estadística Descriptiva e Inferencial, Metodología de la Investigación Científica, entre otras (UABC$\mathrm{FCH}, 1986$ ). También se llevan a cabo eventos de actualización y capacitación docente y estudiantil. El equipo de cómputo en estas aulas también se ha utilizado como herramientas de apoyo a la investigación, porque tanto alumnos como docentes investigadores utilizan estas áreas como apoyo en sus proyectos, además, son comúnmente utilizados para la realización de prácticas y tareas escolares.

Aunado a lo anterior, desde que existe el laboratorio de informática su infraestructura y equipos se han utilizado para apoyar los procesos administrativos de inscripción y reinscripción de su alumnado, cubriendo la función de apoyo a los procesos administrativos.

\section{CREACIÓN DE LA NUEVA BIBLIOTECA DEL SIA}

Desde su creación, la carrera de Ciencias de la Educación contó con una biblioteca que apoyó en todo momento la formación de su alumnado, y aunque en su inicio el acervo bibliográfico fue limitado, ha sido una de las prioridades de las autoridades universitarias. Así lo demuestra el gran desarrollo que ha tenido a lo largo de casi cuatro décadas.

Todo empezó en un aula de clase acondicionada como biblioteca en el salón 203, en donde se contaba con una persona encargada y se daban los servicios básicos de consulta y préstamo de libros, es decir, fundamentalmente se cubriría la función de herramienta didáctica en apoyo a la docencia. Así fue durante algunos años, hasta que al inicio de los años ochenta se construyó el edificio de los 300 y se acondicionaron los salones 308 y 309 a donde se trasladó, ofreciendo los mismos servicios y cubriendo la misma función, pero en un lugar más cómodo y con un acervo más amplio.

Con la creación de la licenciatura en Psicología y la licenciatura en Ciencias de la Comunicación en 1985 y 1986 respectivamente, también se creó la necesidad de un espacio que le brindara el servicio a las tres carreras, razón por la cual se construyó un edificio para que fungiera especialmente como tal. Así surgió el edificio de los 600 , que albergó a la nueva biblioteca con un espacio y acervo bibliográfico mucho más amplio y se adquirieron también algunas bases de datos electrónicas en discos compactos. Como se puede apreciar, ya se utilizaban, además del libro impreso y revistas especializadas, algunos dispositivos tecnológicos como herramientas didácticas y como apoyo a la investigación.

En 2001 se inaugura la Biblioteca de la FCH con instalaciones propias que satisfacen cualitativa y cuantitativamente las necesidades de sus usuarios. El edificio que alberga la biblioteca 
es de dos plantas, con capacidad para atender a un poco más de 350 usuarios de manera simultánea. Además, cuenta con un edificio anexo de una planta para apoyar los servicios de cómputo (laboratorio con capacidad de 40 usuarios) y capacitación (2 salas para 20 y 40 usuarios) (UABC-FCH, 2015). Además de caracterizarse por tener un diseño amplio y modernista, la biblioteca fue construida bajo los estándares establecidos por la UNESCO para este tipo de edificaciones, con la calidad en lo ergonómico, salud y seguridad. Por ello, todas sus áreas cuentan con iluminación tanto natural, a través de ventanas, como artificial, proporcionada por lámparas de neón. Además cuenta con aire acondicionado en todas las instalaciones y puertas de acceso automáticas y manuales, además de puertas de emergencia y alarmas para aviso en caso de siniestro.

Para el apoyo de personas con discapacidad, todos sus accesos son amplios y acordes a las diferentes necesidades que presenten los usuarios. El acceso principal cuenta con rampa; además se cuenta con elevador con capacidad para 6 personas y mobiliario acorde a cada área y servicio que se ofrece, caracterizado por ser ergonómico, estético y en muy buen estado. Los equipos de cómputo disponible para los usuarios suman 86 computadoras disponibles para el acceso a catálogos, bases de datos y otros servicios de información electrónica.

La biblioteca cuenta con 2 salas de lectura con capacidad para 6 usuarios, 2 cubículos de videoconsulta, 1 sala de capacitación con 13 computadoras, 1 laboratorio de cómputo para impartir clase, 1 sala de usos múltiples para exposiciones con capacidad de 30 usuarios, 2 salas anexas de usos múltiples, 1 sala de internet para consulta de información con 25 computadoras con acceso a internet, 1 laboratorio de cómputo para clase, 2 áreas automatizadas de préstamo del acervo bibliográfico, 2 islas con 4 computadoras c/u para consulta en línea del acervo bibliográfico, 14 mesas de trabajo con capacidad de atención a 56 usuarios a la vez, 1 videoteca, 1 hemeroteca, 1 site de telecomunicaciones y 2 áreas de baños (UABC$\mathrm{FCH}, 2015)$.

En la actualidad la biblioteca cuenta con 24,771 títulos y 38,299 volúmenes para apoyar a los diferentes programas educativos que ofrece la Facultad de Ciencias Humanas. Solo para la carrera de educación se cuenta con un acervo especializado en dicha área de 14,009 títulos y 24,091 volúmenes (UABC-DIA, 2016a).

Además de todo esto, en el Sistema UABC se cuenta con 22 bases de datos, que se han convertido en un recurso invaluable para contribuir a una formación actualizada e integral del alumnado, así lo demuestran las estadísticas de uso de las bases de datos en donde se puede observar que durante 2015, la mayoría de los usuarios consultan la base de datos EBSCO con 188,455 consultas de textos, las consultas de los libros de Pearson fueron 1,426 , las consultas totales de los libros de Springer fueron de 19,870 (UABC-DIA, 2016b).

Con este nivel de infraestructura y equipamiento y calidad de servicios de información académica brindados a sus usuarios, en Biblioteca se privilegian las funciones de las TIC como herramientas didácticas, como herramientas de apoyo a la investigación, como objeto de estudio y, así también, como herramientas de apoyo a los procesos administrativos, tanto en la modalidad escolarizada como semiescolarizada.

\section{SURGIMIENTO DE LA MODALIDAD SEMIESCOLARIZADA}

En la Facultad de Ciencias Humanas se cuenta con dos modalidades oficiales de aprendizaje, la escolarizada y la semiescolarizada. El origen de la modalidad semiescolarizada data del surgimiento del Modelo Curricular Flexible por Competencias en el ciclo 2003-2. A la 
par, en la ciudad de Ensenada se ofertaron programas en dicha modalidad, "lo que origina el reconocimiento de nuevas potencialidades en las características de la licenciatura en el marco de las innovaciones propias de sistema formativo pensado en públicos no atendidos hasta el momento en la universidad" (UABC, 2011a: 17).

La modalidad semiescolarizada ha sido muy exitosa y demandada por la naturaleza de su operación. En los Lineamientos Generales para la Operación de la Modalidad Semiescolarizada (UABC, 2011a) se declara que las políticas de flexibilización de la UABC posibilitan la promoción de "modalidades de formación profesional flexibles y menos escolarizadas para así responder a la demanda creciente de educación superior en la entidad, y a su vez atender a un sector de la población, el cual por sus condiciones de vida diferente requiere de espacios de aprendizaje más flexibles" (p. 18). Se buscó con esta modalidad dar respuesta a las demandas sociales y con ello aportar para el fortalecimiento de la educación superior, alineados siempre a la normatividad y política pública vigente. Se dio respuesta a los señalamientos de la Declaración Mundial Sobre la Educación Superior en el Siglo XXI de la UNESCO, específicamente en lo referente a reforzar el desarrollo académico, diversificar el acceso a públicos cada vez más heterogéneos, lograr una difusión universal y con miras a la educación a lo largo de toda la vida (UNESCO, 1998).

A su vez, el Plan Maestro de Educación Superior Abierta y a Distancia, publicado en 2001 , señaló que esta modalidad:

...tiene un efecto multiplicador y enriquecedor en la educación presencial, ya que favorece y fortalece el trabajo en equipo, el aprendizaje basado en la resolución de problemas, las habilidades para la comunicación, la creatividad y la capacidad de innovar. Esto constituye una parte esencial de la estrategia para el fortalecimiento de la educación superior de la nación (ANUIES, 2001: 12).

Por su parte, el modelo educativo de la Universidad Autónoma de Baja California vigente se basaba en cuatro principios: la formación a lo largo de la vida, la educación centrada en el aprendizaje del estudiante, un currículo apoyado en la generación del conocimiento y servicio social, y la formación integral del estudiante; todo ello a través de la flexibilidad en el proceso educativo, un diseño curricular basado en competencias, la vinculación y compromiso social y la colegialidad académica (UABC, 2006). De acuerdo con lo anterior, la integración de la modalidad semiescolarizada requirió el planteamiento de diferentes formas de trabajo académico y administrativo que identificaran áreas de oportunidad para el desarrollo de los profesores y estudiantes.

En los Lineamientos Generales para la Operación de la Modalidad Semiescolarizada, publicados en el Plan de Estudios 2012-2, se establece como objetivos de ésta el integrarla a las Licenciaturas de las Facultades de Ciencias Humanas y Ciencias Administrativas y Sociales como opción formativa no convencional correspondiente al modelo de flexibilización curricular por competencias, así como incorporar personal académico para la instrumentación pedagógica pertinente de dicha modalidad bajo la referencia del modelo educativo de la universidad, considerando las aspiraciones personales de los estudiantes y las necesidades sociales identificadas en el área de conocimiento (UABC-FCH, 2011). Dirigida preponderantemente a la población adulta que cuenta con experiencia laboral, favorece en el estudiante la generación de experiencias autodidactas acrecentando el nivel 
de responsabilidad, participación y compromiso con los aprendizajes, así mismo propicia el desarrollo de propuestas pedagógicas creativas sustentadas en el modelo educativo de la UABC.

Actualmente, las TIC han sido herramienta fundamental en los procesos de comunicación entre profesores y estudiantes y para el sustento de los procesos de aprendizaje, esto porque han permitido eficientar los tiempos presenciales y con mayor énfasis, los no presenciales, al lograr estar en comunicación durante la semana por medios electrónicos. La incorporación de la plataforma de cursos en línea Blackboard como una herramienta institucional de trabajo a distancia ha sido pieza clave en estos procesos. El Plan de Desarrollo actual de la FCH, declara su interés por capacitar particularmente a los profesores que laboran en la modalidad semiescolarizada, en el uso de Blackboard para la impartición de clases.

Como se puede observar, las TIC constituyen elementos fundamentales en el desarrollo de esta modalidad cumpliendo con la función de herramientas de apoyo a la docencia y como contenido $\mathrm{u}$ objeto de estudio.

\section{EL CENTRO DE EDUCACION ABIERTA Y A DISTANCIA (CEAD)}

Desde hace más de dos décadas, en la UABC se han presentado esfuerzos importantes para la incorporación de las TIC en los procesos educativos, pero se puede decir que todos estos esfuerzos fueron aislados, hasta la aparición del CEA en el año 2006:

El Centro de Educación Abierta (CEA) de la $U A B C$, surge de las actividades de la Red de Metodología e Investigación, nace como una comunidad de aprendizaje integrada por académicos de tres de las principales ciudades del Estado de Baja California, en México.
Formados en diferentes disciplinas, acordaron diseñar un programa sobre el tema de metodología de investigación. La dispersión geográfica de su ubicación los obligó a implementar el uso de las tecnologías de Internet y diversos recursos de soporte para poder comunicarse y llevar diversas actividades de colaboración y producción académica en conjunto. Esta experiencia y conocimientos generados los llevó a integrar un Centro cuyas propuestas han logrado convertirse en iniciativas de la institución para la incorporación de TIC en procesos educativos (Lloréns et al., 2009: 226).

El objetivo básico del CEA fue el de brindar servicios institucionales para la administración de cursos en línea, mediante asesoría, capacitación y servicios adicionales sobre tecnologías de información para la docencia que requieran los programas educativos de la UABC (Zamora, 2011). Como parte de la evolución del centro, en septiembre de 2015 se pasó a llamar Centro de Educación Abierta y a Distancia de la UABC (CEAD).

Actualmente el CEAD apoya a la UABC a alcanzar su objetivo de ampliar y diversificar la cobertura con pertinencia y calidad de los servicios de educación superior. El eje de sus iniciativas ha sido la formación y desarrollo de redes de colaboración y cooperación educativa, para fortalecer el diseño de programas utilizando como apoyo las TIC, con diseños bajo un enfoque de aprendizaje distribuido (también conocido como educación a distancia en línea) en beneficio del aprendizaje para promover habilidades académicas, la autonomía y el sentido de la responsabilidad en los alumnos. Además de estar a cargo de la formación docente en competencias tecnológicas y de la evaluación de tecnologías educativas y su 
transferencia para ser incorporados de manera institucional.

En el Acuerdo de Creación del Centro de Educación Abierta y a Distancia de la UABC (2015a) se publicaron las funciones del mismo, tales como:

- Promover la creatividad, conocimiento y experiencia en los aspectos educativo, tecnológico y organizacional, que permitan desarrollar soluciones innovadoras para la pertinencia y calidad del proceso de enseñanza aprendizaje, en modalidades diferentes a la presencial.

- Promover y llevar a cabo investigación sobre temas relevantes en el campo de la educación, relacionados con modalidades educativas apoyadas en las TICC.

- Promover alianzas estratégicas con unidades académicas y dependencias administrativas de la UABC, así como con entidades públicas y privadas para el desarrollo de proyectos.

- Ofrecer cursos y programas educativos, formales y no formales, en modalidades diferentes a la presencial.

- Fomentar la integración y operación de redes de aprendizaje y colaboración, con la participación de líderes y cuerpos académicos, que fortalezcan la capacidad de crear y operar programas educativos de la UABC con apoyo de las TICC.

Como se puede apreciar, la figura del CEAD ha sido parte importante en la integración de las TIC en la educación, tanto en el empleo de ellas por parte estudiantes y profesores, como en lo más complejo de lograr, la integración de las mismas en los procesos de aprendizaje. Así como también uno de los principales promotores del uso de las TIC como herramientas de enseñanza y aprendizaje integradas curricularmente a procesos semiescolarizados y no escolarizados o en línea; como contenido o parte fundamental del objeto de estudio de la carrera y, por último, como herramientas de apoyo a los procesos administrativos.

\section{MODALIDADES NO PRESENCIALES $Y$ MIXTAS}

En la iniciativa siete del Plan de Desarrollo Institucional 2003-2006 se percibe interés genuinoy profundo dela institución porla inclusión de las tecnologías en los procesos educativos. En esta iniciativa, titulada "Tecnologías de la Información y la Comunicación", se aborda la integración de las TIC como una herramienta de trabajo a los procesos académicos sustantivos. Se trataba de promover que las actividades de docencia, investigación, extensión y vinculación incorporaran, en función de su pertinencia, las herramientas de la tecnología informática de la manera más amplia y productiva posible. También se promovió el uso de una plataforma informática y comunicativa en la oferta de cursos y programas educativos pertinentes. En ese momento, se oficializan los trabajos correspondientes al CEA y la plataforma Blackboard.

En este mismo PDI (UABC, 2003) se publicaron seis iniciativas específicas, derivadas de la iniciativa general antes mencionada:

\subsection{Difusión de las TIC.}

7.2. Uso de las TIC como apoyo en la búsqueda de información para el trabajo académico y el desarrollo institucional.

7.3. Capacitación a maestros y estudiantes en el uso de las TIC. 
7.4. Integración de las TIC a los procesos de capacitación del personal.

7.5. Profesionalización de la producción de cursos a distancia.

7.6. Infraestructura y desarrollo tecnológico de soporte y seguridad para las TIC

Encontramos en estas líneas fundamento sólido de la importancia e interés hacia la implementación de las TIC y con ello la diversificacion de modalidades (no presenciales y mixtas).

Por su parte el Plan de Desarrollo Institucional 2007-2010 (2007), presentó la política institucional número diez, encargada de regir lo referente a la infraestructura en el tema de las TIC: "Política institucional 10. Desarrollo equilibrado y operación eficiente de la planta física e infraestructura educativa”. (p.23)

En ella se publicó el gran compromiso de la administración general por equipar y abastecer a las facultades y bibliotecas de la infraestructura necesaria para que la incorporación de las TIC sea una realidad. Así lo determinó el afianzamiento del Sistema de Bibliotecas de la UABC, las tecnologías de la información como apoyo a las funciones sustantivas de la Universidad y el fortalecimiento de la infraestructura de redes y servicios de telecomunicaciones (UABC, 2007).

Por otro lado, el Plan de Desarrollo Institucional de la UABC 2011-2015, en su octava política institucional "Optimización de la infraestructura y equipamiento educativos" (UABC, 2011b: 81), publica la necesaria modernización del equipamiento de apoyo al proceso enseñanzaaprendizaje. Esta iniciativa atiende los requerimientos de las modificaciones de planes de estudio, pues uno de sus objetivos es modernizar el equipo de apoyo al proceso de enseñanza-aprendizaje, en particular el equipo dañado $u$ obsoleto que se utiliza en clase o en los laboratorios de cómputo y, el otro, es el de mantener actualizadas las plataformas de cómputo y redes de la institución.

En el mismo PDI 2011-2015 (UABC, 2011b), en la iniciativa "1.2. Formación pertinente y de buena calidad en respuesta a las necesidades sociales", de la primera política institucional "Impulso a la formación de los alumnos", encontramos algo muy interesante respecto a las modalidades de aprendizaje.

Se trata de la relevancia que se le da a la contribución de la UABC, en cuanto a la atención de la demanda de educación superior con equidad. Son dos los objetivos de esta iniciativa específica: por una parte, ratificar la disposición de la UABC a continuar contribuyendo a atender la creciente demanda de educación superior en Baja California mediante "el impulso a las modalidades de educación semipresencial y a distancia" y, por la otra, "generar mecanismos tanto de acceso a la educación superior como de retención, para población en condiciones de desventaja social" (UABC, 2011b: 64).

Pero es hasta el 2015, con la gestión del Dr. Ocegueda y con el Mtro. Adolfo Soto Curiel en la dirección de la Facultad de Ciencias Humanas, que se promueve de forma intensiva la oferta de asignaturas completamente a distancia o "en línea" con el objetivo de eficientar infraestructura, apoyar a los estudiantes en su formación y se trabaja en cumplimiento de la misión y logro de la visión 2025, el Plan de Desarrollo Institucional 2015-2019 (UABC, 2015b), considera las políticas generales siguientes: 1 . Se asegurará que la ampliación y diversificación de la oferta educativa se sustente en estudios de necesidades del desarrollo social y económico de Baja California. Y 2, se privilegiará la ampliación y diversificación de la oferta educativa a través de la modalidad mixta. 
Es entonces, en el ciclo escolar 2015-2 que se implementan oficialmente las asignaturas completamente en línea, denominándolas formalmente "virtuales", soportadas estas en la plataforma de cursos en línea Blackboard, en asesoría y comunicación constante con el CEAD.

Para el ciclo 2015-2 las asignaturas optativas ofrecidas en la Licenciatura en Ciencias de la Educación en esta modalidad fueron diecisiete, incrementándose la oferta semestre tras semestre. Estas se encuentran distribuidas en los semestres de esta forma:

Tabla 1.

\begin{tabular}{cccc}
\hline & Ciclo 2015- & Ciclo 2016- & Ciclo 2016- \\
Semestre & $\mathbf{2}$ & $\mathbf{1}$ & $\mathbf{2}$ \\
3ero & 3 & 3 & 3 \\
4to & 2 & 2 & 3 \\
5to & 2 & 6 & 3 \\
6to & 2 & 3 & 4 \\
7mo & 4 & 4 & 4 \\
8vo & 3 & 3 & 6 \\
& 16 & 21 & 23 \\
Total & asignaturas & asignaturas & asignaturas \\
\hline
\end{tabular}

Fuente: elaboración propia

Como se puede apreciar, la evolución de la modalidad a distancia ha sido importante $y$ estas acciones han propiciado además, la diversificación de la oferta; la capacitación y habilitación de profesores para trabajar de forma virtual y se ha eficientado el equipamiento y la infraestructura.

\section{IDEAS CONCLUYENTES}

Lo anterior confirma una vez más el gran interés de la UABC, de la Facultad de Ciencias Humanas y de la Licenciatura en Ciencias de la Educación por “....promover alternativas viables para el desarrollo social, económico, político y cultural de la entidad y del país, en condiciones de pluralidad, equidad, respeto y sustentabilidad; y con ello contribuir al logro de una sociedad más justa, democrática y respetuosa de su medio ambiente" (UABC, 2015b: 53). Todo ello mediante la realización de acciones armónicamente relacionadas con las políticas educativas a nivel nacional, y estatal y en donde a las Tecnologías de Información, Comunicación y Colaborativas, se les confiere un papel fundamental para el logro de dichos fines. La Carrera de Ciencias de la Educación de la $\mathrm{FCH}$, sin ser ajena a este contexto, ha logrado mediante el esfuerzo de su comunidad a lo largo de su historia, el reconocimiento de la sociedad como una institución que ofrece servicios educativos de calidad.

\section{REFERENCIAS BIBLIOGRÁFICAS}

Aguerrondo, I. (2006). Estado del arte y orientaciones estratégicas para la definición de políticas educativas en el sector. En La integración de las Tecnologías de la Información y la Comunicación en los Sistemas Educativos. Buenos Aires: IIPEUNESCO Sede Regional Buenos Aires. Recuperado de: http://www. udelas.ac.pa/biblioteca/librospdf/1_ estadodelarte.pdf

ANUIES. (2001). Plan Maestro de Educación Superior Abierta y a Distancia. Líneas estratégicas para su desarrollo. México. Recuperado de: http://sistemas.dti. uaem.mx/sead/anuies-centrosur/pdf/ plan.pdf

Cabero, J. (2007). Las necesidades de las TIC en el ámbito educativo: oportunidades, riesgos y necesidades. Revista de Tecnología y Comunicación Educativas, año 21, núm. 45, 4-19. Recuperado de: http://cursa.ihmc.us/rid=1M92QYFT52BBGPTG-1QT0/julio\%20cabero.pdf 
Consejo Federal de Educación. (2010). Las políticas de inclusión digital educativa: El Programa Conectar Igualdad. Recuperado de: http://www.me.gov.ar/ consejo/resoluciones/res10/123-10_01. pdf

Fernández, A. (2011). La inclusión de las tecnologías de la comunicación e información como herramientas clave en la modernización de la enseñanza en América Latina y el Caribe. Boletín IESAIC Informa de Educación Superior, 217. Recuperado de: http://www.iesalc. unesco.org.ve

Ferreiro, R. F. y De Napoli, A. (2008). Más allá del salón de clases: los nuevos ambientes de aprendizaje. Revista Complutense de Educación, 19 (2), 333-346. Recuperado de: https://revistas.ucm. es/index.php/RCED/article/viewFile/ RCED0808220333A/15480

Gobierno de la República. (2001). Plan Nacional de Desarrollo 2001-2006. México: Autor. Recuperado de: http://www.diputados. gob.mx/LeyesBiblio/compila/pnd.htm

Gobierno de la República. (2007). Plan Nacional de Desarrollo 2007-2012. México: Autor. Recuperado de: http://pnd.calderon. presidencia.gob.mx/pdf/PND_20072012.pdf

Gobierno de la República. (2013). Plan Nacional de Desarrollo 2013-2018. México: Autor. Recuperado de: http://www.snieg.mx/ contenidos/espanol/normatividad/ MarcoJuridico/PND_2013-2018.pdf

Gobierno del Estado de BC. (2014). Plan Estatal de Desarrollo 2014-2019. Recuperado de: http://www.bajacalifornia.gob.mx/ portal/gobierno/ped/ped.jsp
Herrera, Y. (s.f.). Propuesta de un modelo de Integración curricular de las Tics. Universidad Tecnológica Metropolitana (UTM).

Lloréns, L., Figueroa, C., Espinosa, Y., Perezchica, E., Gaona, T., Sepúlveda, J. y Lizalde, F. (2009). Creación de comunidades docentes de aprendizaje colaborativo para la difusión de estrategias de educación abierta y a distancia apoyadas en tecnologías de información, comunicación y colaboración. Recursos digitales para el Aprendizaje (pp. 223-232). Yucatán, México: Ediciones Universidad Autónoma de Yucatán.

Nosiglia, M. C. y Barboza, L. (2013). Las políticas de inclusión de tecnologías en la enseñanza: una comparación entre los casos de Argentina y Uruguay. Revista Latinoamericana de Educación Comparada, año 4, núm. 4, 25-40. Recuperado de: http://www.saece.com. ar/relec/revistas/4/ mon2.pdf

Pariente Alonso, F. J. (2005). Hacia una auténtica integración curricular de las tecnologías de la información y comunicación. En Revista Iberoamericana de Educación. No. 36. Organización de Estados Iberoamericanos para la Educación, la Ciencia y la Cultura (OEI). España.

Parra, E. y Pincheira, R. (s.f.). Integración curricular de las TIC. Recuperado de: https://www.oas.org/cotep/GetAttach. aspx?lang $=$ es $\&$ cld $=412 \&$ aid $=707$

Sánchez, J. (2003). Integración Curricular de TICs: Concepto y Modelos. Revista Enfoques Educacionales, 5 (1), 5165. Recuperado de: http://www.facso. uchile.cl/publicaciones /enfoques/07/ Sanchez_IntegracionCurricularTICs.pdf 
SEP. (2001). Programa Nacional de Educación 2001-2006. México: Autor. Recuperado de: www.oei.es/historico/quipu/mexico/ Plan_educ_2001_2006.pdf

SEP. (2007). Programa Sectorial de Educación 2007-2012. México: Autor. Recuperado de: www.oei.es/historico/quipu/mexico/ programa_sectorial_educacion_mexico. pdf

SEP. (2013). Programa Sectorial de Educación 2013-2018. México: Autor. Recuperado de: http://www.sep.gob.mx/work/ models/sep1/Resource/4479/4/images/ PROGR AMA_SECTORIAL_DE_ EDUCACION_2013_2018_WEB.pdf

Sistema Educativo Estatal. (2015). Programa de Educación de Baja California 2015-2019. Recuperado de: http:// www.educacionbc.edu.mx/see/ programasectorial/PEBC20152019.pdf

UNESCO. (1998). Declaración Mundial Sobre la Educación Superior en el Siglo XXI: Visión y Acción. París, Francia: Autor. Recuperado de: http://www.unesco.org/ education/educprog/ wche/declaration_ spa.htm

UABC. (2003). Plan de Desarrollo Institucional 2003-2006. Mexicali: Autor. Recuperado de: http://www.uabc.mx/planeación/ pdi/2003-2006/PDI.PDF

UABC. (2006). Modelo Educativo de la Universidad Autónoma de Baja California. Mexicali, México: Autor (Cuadernos de Planeación y Desarrollo Institucional.

UABC. (2007). Plan de Desarrollo Institucional 2007-2010. Mexicali: Autor. Recuperado de: http://www.uabc.mx/planeación/ pdi/2007-2010/PDI.pdf
UABC. (2011). Lineamientos Generales para la Operación de la Modalidad Semiescolarizada UABC. Facultad de Ciencias Humanas, Campus Mexicali y Facultad de Ciencias Administrativas y Sociales, Campus Ensenada, B.C.

UABC. (2011). Plan de Desarrollo Institucional 2011-2015. Mexicali: Autor. Recuperado de: http://www.ens.uabc.mx/sege/ PDI_2011_2015.pdf

UABC. (2015). Acuerdo de Creación del Centro de Educación Abierta y a Distancia de la UABC. Mexicali, México: Autor. Recuperado de: http://sriagral.uabc.mx/ Externos/AbogadoGeneral/ index_htm_ files/39.pdf

UABC. (2015). Plan de Desarrollo Institucional 2015-2019. Mexicali: Autor. Recuperado de: http://www.uabc.mx/planeacion/ pdi/2015-2019/PDI-2015-2019.pdf

UABC-DIA. (2016). Estadística total de títulos y ejemplares por disciplinas que atiende el DIA 2015. (Manuscrito no publicado). Departamento de Información Académica de la Facultad de Ciencias Humanas, DIA. Mexicali, B. C., México.

UABC-DIA. (2016). Informe de Biblioteca 2015. (Manuscrito no publicado). Departamento de Información Académica de la Facultad de Ciencias Humanas, DIA. Mexicali, B. C., México.

UABC-ECE. (1983). Compendio Informativo de la Escuela de Ciencias de la Educación 1977-1982. (Manuscrito no publicado). Mexicali, B.C. México.

UABC-FCH. (1986). Plan de Estudios de la Licenciatura en Ciencias de la Educación 1986-2. (Manuscrito no publicado). Mexicali, B.C. México. 
UABC-FCH. (2011). Plan de Estudios de la Licenciatura en Ciencias de la Comunicación 2011. Modificación y Homologación de Planes de Estudio en la Licenciatura en Ciencias de la Comunicación: Facultad de Ciencias Administrativas y Sociales, Facultad de Ciencias Humanas y Facultad de Humanidades. UABC: Autor. Recuperado de http://fch.mxl.uabc.mx/ wp-content/uploads/2015/04/plan-deestudios-comunicacion-2011-1.pdf

UABC-FCH. (2012). Plan de Estudios de la Licenciatura en Ciencias de la Educación 2012-2. Mexicali: Autor. Recuperado de: http://fch.mxl.uabc.mx/ wp-content/uploads/2015/04/ proyectomodificacion-educacion.pdf

UABC-FCH. (2015). Informe para la Acreditación de la Licenciatura en Ciencias de la Comunicación. (Manuscrito no publicado). Categoría 6: Servicios de apoyo; Indicadores 63 y 64 . Mexicali, B. C., México.

Zamora, M. (2011). La capacitación de alumnos y la formación docente, claves para la incorporación de TIC en el aula. Caso de éxito FCQU, de la UABC. Tijuana: UABC. Recuperado de: http://recursos. portaleducoas.org/publicaciones/lacapacitaci-n-de-alumnos-y-la-formacin-docente-claves-para-la-incorporaci-nde-tic 